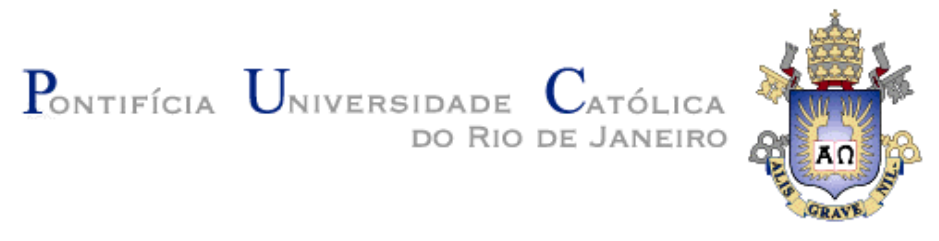

Ricardo Augusto Boiteux Mendes Leal

Teste Funcional baseado em Modelos Gramaticais

Dissertação de Mestrado

Dissertação apresentada como requisito parcial para obtenção do título de Mestre pelo Programa de PósGraduação em Informática da PUC-Rio.

Orientador: Arndt von Staa

Rio de Janeiro

Março de 2008 


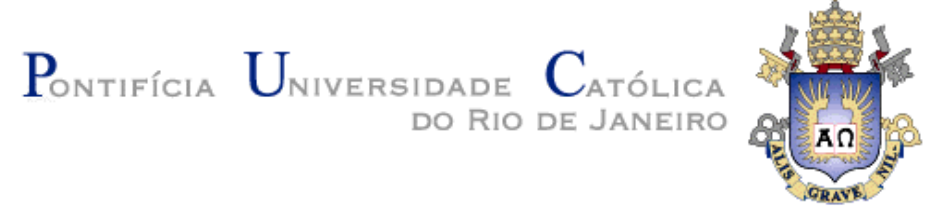

Ricardo Augusto Boiteux Mendes Leal

\section{Teste Funcional baseado em Modelos Gramaticais}

Dissertação apresentada como requisito parcial para obtenção do título de Mestre pelo Programa de PósGraduação em Informática da PUC-Rio. Aprovada pela Comissão Examinadora abaixo assinada.

Arndt von Staa

Orientador

PUC-Rio

Carlos José Pereira de Lucena

PUC-Rio

Renato Fontoura de Gusmão Cerqueira

PUC-Rio

Noemi de La Rocque Rodriguez

PUC-Rio

José Eugenio Leal

Coordenador Setorial do Centro Técnico Científico - PUC-Rio

Rio de Janeiro, 17 de março de 2008 
Todos os direitos reservados. É proibida a reprodução total ou parcial do trabalho sem autorização da universidade, do autor e do orientador.

Ricardo Augusto Boiteux Mendes Leal

Graduado engenheiro de computação pela PUC-Rio em 1998. Desde então, atuou na iniciativa privada como analista de sistemas, consultor de tecnologia de informação e sócio de empresas de tecnologia e desenvolvimento de software. Em 2006, ingressou no curso de Pós-Graduação em Informática da PUC-Rio. Hoje, retorna à iniciativa privada como Engenheiro de Software.

Ficha Catalográfica

Leal, Ricardo Augusto Boiteux Mendes

Teste funcional baseado em modelos gramaticais / Ricardo Augusto Boiteux Mendes Leal; orientador: Arndt von Staa. -2008.

$132 \mathrm{f} . ; 30 \mathrm{~cm}$

Dissertação (Mestrado em Informática) - Pontifícia Universidade Católica do Rio de Janeiro, Departamento de Informática.

Inclui bibliografia.

1. Informática - Teses. 2. Gramática. 3. Caso de teste. 4. Teste baseado em modelo. 5. Teste funcional. 6. Teste de software. I. Staa, Arndt von. II. Pontifícia Universidade Católica do Rio de Janeiro. Departamento de Informática. III. Título.

CDD: 004 
Eu costumo classificar as pessoas em dois grupos distintos: aqueles que procuram problemas e aqueles que procuram soluções.

Dedico este trabalho a todos do segundo grupo que, com esforço, imaginação e perseverança, superam desafios, mesmo quando estes parecem impossíveis. Se não fossem estas pessoas, esta vida não teria o menor sentido. 


\section{Agradecimentos}

Agradeço a Deus pela saúde, força, imaginação e perseverança, que permitiram realizar este trabalho e superar os desafios encontrados ao longo de minha vida.

Agradeço aos meus pais Antonio e Ana pelo seu incomensurável apoio e sua preciosa dedicação, estando sempre presentes quando necessário e tendo paciência para compreender os momentos em que estive distante de seu convívio.

Agradeço a minha mãe Myrian (in memoriam) por ter me propiciado esta vida maravilhosa e uma bela família sem a qual não consigo me imaginar.

Agradeço aos meus irmãos Toninho e Sandra pelo incentivo dado em todos os momentos difíceis ao longo desta e de outras trajetórias.

Agradeço aos meus amigos, em particular André Marins, Luiz Marques, Eduardo Fonseca, Daniela Brauner e Márcio Aguiar, pelo seu companheirismo, sua sinceridade, seu respeito, seus conselhos e sua presença marcante nestes anos.

Agradeço ao professor Arndt von Staa pela sua valiosa orientação, pelos conhecimentos adquiridos em nossas conversas e pelo seu excelente humor.

Agradeço aos professores Arndt von Staa e Noemi Rodriguez pela confiança em mim depositada e pela oportunidade a mim concedida via suas cartas de referência para o curso de Mestrado.

Agradeço ao Laboratório de Engenharia de Software (LES), representado pelo professor Carlos Lucena, por ter me acolhido como seu membro integrante e por ter propiciado um ótimo espaço para troca de idéias e de conhecimento entre seus participantes.

Agradeço ao Departamento de Informática (DI) e à Pontifícia Universidade Católica do Rio de Janeiro, representados por seus professores e funcionários, pelo excelente curso de Mestrado oferecido, e à Coordenação de Aperfeiçoamento de Pessoal de Nível Superior (Capes) pelo financiamento de minha pesquisa. 


\section{Resumo}

Leal, Ricardo Augusto Boiteux Mendes; Staa, Arndt von. Teste Funcional baseado em Modelos Gramaticais. Rio de Janeiro, 2008. 132p. Dissertação de Mestrado - Departamento de Informática, Pontifícia Universidade Católica do Rio de Janeiro.

O teste funcional de software é um desafio enfrentado há muito tempo por desenvolvedores. A complexidade crescente de sistemas computacionais torna esse desafio ainda maior. Uma tendência apontada como possível solução deste problema é o uso de teste baseado em modelos. Inspirada neste paradigma, esta dissertação retrata uma pesquisa sobre o uso de gramáticas como modelos de teste funcional. Modelos gramaticais podem capturar conceitos e comportamentos de um sistema e de seu ambiente usando um nível de abstração de acordo com o objetivo de teste. Eles também podem ser aplicados para descrever casos de teste funcional e guiar a execução de casos de teste gerados num sistema em teste. $\mathrm{O}$ resultado desta execução, representado na forma de um veredicto, revela a conformidade do sistema com seus requisitos e especificações. Para explorar o potencial dos modelos gramaticais, este trabalho definiu uma maneira sistemática de gerar e executar massas de teste. Esta solução permitiu a programação de diferentes estratégias de teste. Ela também facilitou a adaptação dos testes a mudanças de requisitos e promoveu o reuso dos testes existentes. Como efeito colateral deste estudo, um processo de teste funcional foi desenvolvido e a arquitetura de apoio aqui introduzida pode ser reutilizada ou estendida por futuras soluções de teste funcional.

\section{Palavras-chave}

Gramática; caso de teste; teste baseado em modelo; teste funcional; teste de software. 


\section{Abstract}

Leal, Ricardo Augusto Boiteux Mendes; Staa, Arndt von. Grammar Model-Based Functional Test. Rio de Janeiro, 2008. 132p. MSc. Dissertation - Departamento de Informática, Pontifícia Universidade Católica do Rio de Janeiro.

Software functional test is a challenge faced by developers for a long time. The growing complexity of computing systems turns this challenge even greater. Model-based testing is a trend pointed out by the academia and the industry as a possible solution to this matter. Inspired by this paradigm, this dissertation depicts a research made on the use of grammars as functional test models. Grammar models can capture concepts and behaviors of a system and its environment at a level of abstraction according to the test goal. They also can be applied to describe functional test cases and guide the execution of the generated test cases against a system under test. The result of this execution, represented as a verdict, reveals the system conformity with its requirements and specifications. In order to explore grammar models potential, this work defined a systematic way to generate and execute a mass of tests. This solution allowed the implementation of different test strategies. It also assisted test adjustment to requirements change and promoted existing tests reuse. As a side-effect of this study, a functional test process was developed and the supporting architecture introduced here may be reused or extended by future functional test solutions.

\section{Keywords}

Grammar; test case; model-based testing; functional testing; software testing. 


\section{Sumário}

1 Introdução 15

1.1. Definição do Problema 16

$\begin{array}{ll}\text { 1.2. Solução Proposta } & 17\end{array}$

1.3. Objetivos 19

1.4. Resultados 20

1.5. Organização da Dissertação 22

2 Contexto de Teste de Software 23

2.1. Terminologia de Teste 24

2.2. Processo de Teste 26

2.3. Escopo e Seleção de Teste 28

2.4. Teste Funcional 29

2.4.1. Técnicas de Teste Funcional 29

2.4.2. Características de uma Ferramenta de Teste Funcional 32

2.5. Automação do Teste 34

2.5.1. Níveis de Automação de Teste 34

2.5.2. Geração Automática de Dados de Teste 35

2.5.3. Gerador de Teste Baseado em Sintaxe 36

2.6. Teste Baseado em Modelo 38

2.6.1. Características do Modelo de Teste 38

2.6.2. Taxonomia de Teste Baseado em Modelo 39

2.6.3. Processo de Teste Baseado em Modelo 40

2.6.4. Níveis de Concretude de Teste 43

2.7. Teste Dirigido por Modelo 44

2.8. Desafios 46

2.8.1. Desafios à Modelagem 46

2.8.2. Desafios à Automação 47

$\begin{array}{ll}\text { 2.8.3. Desafios à Eficiência } & 47\end{array}$

3 Modelos Gramaticais 48 
3.1. Metagramática e Gramáticas de Teste 49

3.2. Sintaxe das Gramáticas de Teste 50

3.2.1. Definições 52

3.3. Semântica das Gramáticas de Teste 54

3.4. Trabalhos Relacionados 54

3.4.1. Sistemas Baseados em Gramáticas 54

3.4.2. Desenvolvimento de Software com Abordagem Gramatical 55

3.4.3. Projeto de um Metamodelo Executável Orientado por Gramática 56

3.4.4. Modelos Independentes e Dependentes de Plataforma 57

3.5. Modelos de Teste 57

3.5.1. Modelo Estrutural do Sistema em Teste 59

3.5.2. Modelo Comportamental do Sistema em Teste 62

3.5.3. Modelo de Casos de Teste 64

3.5.4. Modelo do Ambiente de Teste 68

4 Teste Funcional $\quad 72$

4.1. Processo de Teste Funcional 73

4.1.1. Fases e Atividades $\quad 74$

4.1.2. Exemplo 76

4.2. Preliminares $\quad 77$

4.2.1. Identificar Requisitos e Documentos de Especificação 78

4.2.2. Definir Escopo de Avaliação 79

4.3. Modelagem do Sistema em Teste 82

4.3.1. Especificar Estrutura 83

4.3.2. Especificar Comportamentos 86

4.4. Seleção do Cenário de Teste 88

4.4.1. Definir Critério de Seleção de Teste 91

4.4.2. Especificar Casos de Teste 92

4.4.3. Especificar Ambiente de Teste 95

4.4.4. Gerar Cenário de Teste 97

4.5. Execução e Avaliação do Cenário de Teste 98

4.5.1. Executar Cenário de Teste 100

4.5.1.1. Executar Caso de Teste 102 
5 Estudo de Caso 105

5.1. Preliminares 106

5.2. Modelagem do Sistema em Teste 107

5.3. Seleção do Cenário de Teste 110

5.4. Execução e Avaliação do Cenário de Teste 117

5.5. Resultados 118

6 Discussão 120

6.1. Teste Baseado em Gramática e Outras Abordagens de Teste $\quad 120$

6.2. Contribuições 123

7 Conclusões $\quad 125$

7.1. Análise do Trabalho 126

7.2. Trabalhos Futuros 128

8 Referências Bibliográficas 130 


\section{Lista de figuras}

Figura 1 - Processo de Teste Funcional 20

Figura 2 - Gramática de Teste; Modelo Gramatical;

Modelo Computacional; Sistema 21

Figura 3 - Processo de Teste 26

Figura 4 - Exemplo de Teste Randômico 30

Figura 5 - Exemplo de Teste de Partições Equivalentes 30

Figura 6 - Exemplo de Teste de Valores de Contorno 31

Figura 7 - Exemplo de Teste baseado em Tabelas de Decisão 31

Figura 8 - Exemplo de Teste de Mutação 32

Figura 9 - Interação: Ferramenta e Sistema em Teste

(Adaptação: Andrea, 2007) 33

Figura 10 - Níveis de Automação de Teste (Tradução: Gao et al., 2003) 35

Figura 11 - Exemplo de Notação - BNF 36

Figura 12 - Exemplo de Gramática de Atributos 37

Figura 13 - Processo de Teste Baseado em Modelo

(Adaptação: Utting et al., 2006) 40

Figura 14 - Extensão do Primeiro Passo do Metaprocesso de Teste 41

Figura 15 - Extensão do Segundo Passo do Metaprocesso de Teste 42

Figura 16 - Extensão do Terceiro Passo do Metaprocesso de Teste 42

Figura 17 - Teste de Dirigido por Modelos (Adaptação: Dai, 2004) 45

Figura 18 - Diagrama de Classes da Metagramática de Teste 53

Figura 19 - Exemplo de Sistema em Teste 58

Figura 20 - Representação do Modelo Estrutural 60

Figura 21 - Representação do Modelo Comportamental 63

Figura 22 - Modelo Completo do Sistema em Teste 63

Figura 23 - Representação do Modelo de Casos de Teste 67

Figura 24 - Representação do Modelo de Ambiente de Teste 69

Figura 25 - Representação do Cenário de Teste $\quad 71$

Figura 26 - Processo de Teste Funcional 75

Figura 27 - Fase: Preliminares 77 
Figura 28 - Atividade: Identificar Documentos de Especificação de Requisitos 78

Figura 29 - Exemplo: Documentos do Sistema de Contas Bancárias 79

Figura 30 - Atividade: Definir Escopo de Avaliação 79

Figura 31 - Exemplo: Diagrama de Seqüência da Transferência entre Contas 80

Figura 32 - Exemplo: Documentação da Interface Pública de Conta Bancária 81

Figura 33 - Exemplo: Diagrama de Classes com foco na Conta Bancária 81

Figura 34 - Fase: Modelagem do Sistema em Teste 82

Figura 35 - Atividade: Especificar Estrutura $\quad 84$

Figura 36 - Arquitetura de Representação da Estrutura do Sistema $\quad 84$

Figura 37 - A Dinâmica de Representação da Estrutura do Sistema 85

Figura 38 - Atividade: Especificar Comportamentos 86

Figura 39 - Arquitetura de Representação dos Comportamentos do Sistema 87

Figura 40 - A Dinâmica de Representação dos Comportamentos do Sistema 87

Figura 41 - Fase: Seleção do Cenário de Teste $\quad 89$

Figura 42 - Atividade: Definir Critério de Seleção de Teste 91

Figura 43 - Arquitetura de Estratégia de Teste 92

Figura 44 - Atividade: Especificar Casos de Teste 93

Figura 45 - Arquitetura de Representação dos Casos de Teste 94

Figura 46 - A Dinâmica de Representação dos Casos de Teste 94

Figura 47 - Atividade: Especificar Ambiente de Teste 95

Figura 48 - Arquitetura de Representação do Ambiente de Teste 96

Figura 49 - A Dinâmica de Representação do Ambiente de Teste 96

Figura 50 - Atividade: Gerar Cenário de Teste 97

Figura 51 - Arquitetura de Geração de Cenário de Teste 98

Figura 52 - Fase: Execução e Avaliação do Cenário de Teste 99

Figura 53 - Atividade: Executar Cenário de Teste 101

Figura 54 - Arquitetura de Execução de Teste 101

Figura 55 - A Dinâmica de Execução de Teste 102

Figura 56 - Atividade: Gerar Veredicto de Teste 103

Figura 57 - Arquitetura de Julgamento de Teste 103

Figura 58 - A Dinâmica de Julgamento de Teste 104

Figura 59 - Extensão do Gerador de Teste. 113

Figura 60 - Extensão do Executor de Teste. 117 
Figura 61 - Extensão do Julgamento de Teste.

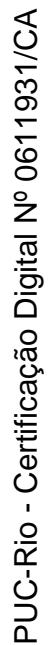




\section{Lista de tabelas}

Tabela 1 - Metagramática de Teste $\quad 51$

Tabela 2 - Gramática de Estrutura $\quad 59$

Tabela 3 - Modelo Estrutural do Exemplo 61

Tabela 4 - Gramática de Comportamento 62

Tabela 5 - Modelo Comportamental do Exemplo 64

Tabela 6 - Gramática de Casos de Teste 65

Tabela 7 - Modelo de Casos de Teste do Exemplo 68

Tabela 8 - Gramática de Ambiente de Teste $\quad 68$

Tabela 9 - Modelo de Ambiente de Teste do Exemplo 70

Tabela 10 - Principais Questões do Processo de Teste. 73

Tabela 11 - Exemplo: Modelo Estrutural $\quad 85$

Tabela 12 - Exemplo: Modelo Comportamental $\quad 88$

Tabela 13 - Exemplo: Modelo de Caso de Teste 95

Tabela 14 - Exemplo: Modelo de Ambiente 97

Tabela 15 - Modelo Estrutural do Estudo de Caso 108

Tabela 16 - Modelo Comportamental do Estudo de Caso 109

Tabela 17 - Modelo de Caso de Teste do Estudo de Caso - Primeiro Critério 111

Tabela 18 - Modelo de Caso de Teste do Estudo de Caso - Segundo Critério 111

Tabela 19 - Modelo de Ambiente do Estudo de Caso 112

Tabela 20 - Código Gerado 116 